\title{
A OBSERVATIONAL STUDY ON CYTOKERATIN 5/6 EXPRESSION IN BREAST CARCINOMA PATIENTS AND CORRELATED WITH HORMONAL STATUS.
}

\section{Pathology \\ Rubaiya Ahmad \\ MBBS, MD, Tutor Dept of Pathology, Sri Krishna Medical Collage Muzaffarpur, Bihar,} India.

Syed Meraj Imam*

MBBS, DORL ENT, J.J.M Medical College, Medical College Rd, MCC B Block, Kuvempu Nagar, Davanagere, Karnataka 577004, India*Corresponding Author

Debarshi Jana

IPGMER and SSKM Hospital, Kolkata.

\begin{abstract}
INTRODUCTION Breast cancer is the second most common cause of morbidity and mortality worldwide in women. They vary remarkably in terms of clinical presentation, history, behaviour and genetic characteristics. There has been a significant increase in overall incidence of breast cancers especially so in the developing countries attributable mainly to globalization causing adaptation of western life style and improved access to diagnostic modalities. OBJECTIVE OF THE RESEARCH Study of cytokeratin 5/6 expression in proliferative, preinvasive and malignant lesions of breast. ER/PR expression in proliferative, preinvasive and malignant lesions of breast. Correlation of data using appropriate statistical methods. METHODOLOGY Patients attending the Dept of Pathology, Sri Krishna Medical Collage Muzaffarpur, Bihar with epithelial breast tumor. RESULT AND CONCLUSION We found that cytokeratin 5/6 expression in Breast Carcinoma was significantly associated with high grade of the tumour. cytokeratin 5/6 expression was significantly associated with advanced stage of the tumour. It was found that cytokeratin $5 / 6$ expression was significantly correlated with ki67 in Breast Carcinoma patients. Hormonal receptor like ER and PR were was significantly associated with cytokeratin 5/6 expression tumour. Triple negative Breast Carcinoma patient was significantly associated with high grade of cytokeratin $5 / 6$ expression tumour.
\end{abstract}

\section{KEYWORDS}

Cytokeratin, er/pr status , proliferative, preinvasive, malignant and breast

\section{INTRODUCTION}

Breast cancer is the second most common cause of morbidity and mortality worldwide in women. They vary remarkably in terms of clinical presentation, history, behaviour and genetic characteristics. There has been a significant increase in overall incidence of breast cancers especially so in the developing countries attributable mainly to globalization causing adaptation of western life style and improved access to diagnostic modalities. Immunohistochemistry has been an important tool in the molecular diagnosis of breast cancer and is efficiently used for differentiating the usual ductal hyperplasia from DCIS(Ductal carcinoma in status), distinguishing invasive carcinomas from preinvasive ones,ruling out microinvasions, identifying breast cancer histological subtyping and molecular phenotype along with a role in estimating prognosis and predicting the therapeutic response.Normal breast gland contains 3 types of epithelial cells: luminal, myoepithelial and stem cells. Myoepithelial cells and luminal cells can be distinguished by their differential cytokeratin expression patterns. Myoepithelial cells typically express CK 5/6 while luminal cells express $\mathrm{Ck} 8 / 18$. $^{2}$ Usual ductal hyperplasia arises from proliferation of luminal cells with stem cell properties while atypical ductal hyperplasia and low grade ductal carcinoma in situ from more differentiated luminal cells. Myoepithelial markers surround the normal benign breast glands and are deficient in invasive carcinomas. Genomic instability is thought to be the underlying mechanism by which cells acquire genetic alterations eventually leading to cancer. Especially in breast cancer, the evidence has in recent years been mounting that the acquisition of genetic aberrations detectable by modern cytogenetic techniques does not happen in an anarchic fashion. Rather, multiple, partially parallel pathways seem to exist, each of them associated with a specific cellular morphology and/or tumor architecture. This has led to the proposal of a morphologybased, cytogenetic progression model from normal to in situ and invasive breast cancer ${ }^{3}$. In this model, the loss of $16 \mathrm{q}$ seems to be the most important step associated with tumor proliferation rate, independent of the rate of cytogenetic instability. Nevertheless, the expected differences in protein expression patterns associated with the cytogenetic events in these pathways remained unclear. In addition, studies on carcinogenesis pathways have to take into account features of presumed precursor cells. Our own studies pointed to changing cytokeratin expression patterns within the physiologic maturation and differentiation of the normal female breast ${ }^{4}$. The aim of this study was therefore to relate patterns of cytogenetic changes reflected by comparative genomic hybridization (CGH) with protein expression patterns assessed on tissue microarrays (TMAs) in relation to the different precursor cells in breast carcinogenesis.
Previous studies on breast cancer classified the disease into different groups based on immunohistochemistry (IHC) profile of estrogen receptor (ER), progesterone receptor (PR) and human epidermal growth factor receptor (Her2/neu) expression, positive (+ve) and/or negative $(-\mathrm{ve})$, which are: ER+PR+HER2+, ER+PR+HER2-, ERPR-HER2+, ER-PR-HER2-, ER+PR-HER2+, ER+PR-HER2-, ER-PR+HER2+, and ER-PR+HER2-. Recent attention has been directed at triple-negative breast cancer (TNBC) (ER-PR-HER2-) and triple-positive breast cancer (TPBC) (ER+PR+HER2+). TNBC is a burning concept worldwide due to unresponsiveness toward effective clinical therapies in comparison to the other types of breast carcinoma. While TPBC provides the significant prognostic information which imparts guidance of response to targeted and proven therapy; for example, endocrine and trastuzumab therapy for tumors expressing ER/PR and Her2/neu. Many studies have reported that tumors expressing triple-negative and triple-positive expression of ER, PR, and Her2/neu receptors were found in basal- and luminallike breast carcinoma, respectively. Further, basal and luminal carcinomas were observed inconsistent association with expression of cytokeratin (CK) 5,14 , and $8 / 18$, respectively, in most of the studies.CK is epithelia-specific intermediate filament proteins, which are expressed in a tissue-specific manner. CK tumor markers can accurately predict disease status before conventional methods and offer a simple, non-invasive, cheap, and reliable tool for more efficient management. CK also plays promising role in vaccination trial and in understanding cellular processes such as apoptosis, mitosis, cell cycle progression, and cell signaling, etc ${ }^{5}$. The aim of this study was to analyze the CK5, 14, and 8/18 markers in breast tissue and to determine the correlation between hormone receptor status (ER, PR, and Her2/neu) along with clinic-pathological factors

1. Study of cytokeratin $5 / 6$ expression in proliferative, preinvasive and malignant lesions of breast.

2. Study of ER/PR expression in proliferative, preinvasive and malignant lesions of breast

\section{METHODOLOGY}

a) Study / Experiment design: Descriptive cross sectional study. b) Study setting: Patients attending the Dept of Pathology, Sri Krishna Medical Collage Muzaffarpur, Bihar with epithelial breast tumor. h) Inclusion criteria:

The specimens of breast biopsies sent to the department of pathology by modified radical mastectomy, truecutbiopsy, lumpectomy etc.

\section{RESULT AND DISCUSSION}

In our study, 6(14.6\%) patients were $\leq 40$ years old, $16(39.0 \%)$ patients were 41-50 years old, 12(29.3\%) patients were 51-60 years old and 
$7(17.1 \%)$ patient were $61-70$ years old. In our study, $17(41.5 \%)$ patients were Left Side and 24(58.5\%) patient were Right Side. In our study, 2(5.6\%) patients were T1N0MX, 15(41.7\%) patients were T2N0MX, 6(16.7\%) patients were T2N1MX, 2(5.6\%) patients were T2N2MX, 2(5.6\%) patients were T3N0MX, 5(13.9\%) patients were T3N1MX, 1(2.8\%) patients were T3N2MX, 2(5.6\%) patients were T4aN1MX and $1(2.8 \%)$ patient were T4bN2MX. In our study, 2(5.6\%) patients were S1 stage, 23(63.9\%) patients were S2 stage and $11(30.6 \%)$ patient were S3 stage. In our study, 2(4.9\%) patients were $\mathrm{ADH}, 2(4.9 \%)$ patients were DCIS, $3(7.3 \%)$ patients were IBC-NST with medullary differentiation, $28(68.3 \%)$ patients were IDCNOS, 1(2.4\%) patients were Lobular Carcinoma, 2(4.9\%) patients were MC, 2(4.9\%) patients were Metaplastic CA and 1(2.4\%) patient were UDH. In our study, 2(5.6\%) patients were Grade I, 23(63.9\%) patients were Grade II and 11(30.6\%) patient were Grade III. In POSITIVE study, $30(73.2 \%)$ patient had ER status.

Jana D et al ${ }^{5}$ (2012) showed that it is necessary to study the utility of HER-2/neu as a prognostic factor in breast cancer survival. However, there have not been detailed studies in this respect with the breast cancer patients of Eastern India. There was statistically significant association between survival pattern for 5 years and the HER-2/neu status $(\mathrm{p}=0.00001)$. Better survival was observed for the patients with HER-2/neu negative tumors $67(100 \%)$ compared to HER-2/neu positive tumors $7(36.8 \%)$. There is strong interaction between survival and HER-2/neu expression of breast cancer patients. Thus the patients with HER-2/neu positive tumors need to be treated aggressively. Our study also showed that $10(24.4 \%)$ patients had positive Her 2 neu. Association of Her 2 neu vs CYTOKERATIN 5/6 was not statistically significant $(\mathrm{p}=0.1321)$.

Maeda I et al ${ }^{6}(2018)$ showed that to use immunohistochemistry to differentiate solid papillary carcinoma in situ from intraductal papilloma with usual ductal hyperplasia (IPUDH). 6(14.6\%) patient had Cytokeratin 5/6. Three types of high-molecular-weight cytokeratins (CKs) - CK5/6, CK14, and CK34betaE12 - were targeted. Immunohistochemical staining with CK34 beta E12 antibody produced scores of $1-3$ in all patients with solid papillary carcinoma and 3-5 in all patients with IPUDH. In tissues from patients with IPUDH, significantly more cells were stained with CK34betaE12 than CK5/6 (p $<0.05)$ or CK14 $(\mathrm{p}<0.05)$.

Fadare $\mathrm{O}$ et al ${ }^{7}(2007)$ found that there are 2 well-recognized cell populations lining the mammary duct system: the epithelial cells lining the lumen and the myoepithelial cells surrounding them. 5(13.9\%) patient had HER2-positive (non-luminal), 1(2.8\%) patient had Luminal A-like, 19(52.8\%) patient had Luminal A-like, 3(8.3\%) patient had luminal B-like HER 2+VE, 3(8.3\%) patient had Luminal B-like( HER2-NEG) and 5(13.9\%) patient had Triple-negative.There seems to be a phenotypic and biologic spectrum of basal-like or myoepithelial-type carcinomas, just as there is a wide range among tumors of luminal epithelial derivation/differentiation. It is critical to promote lucid morphologic definitions of the molecular subtypes, if this information is intended for use in targeted therapies and patient management.

Van De Rijn M et al ${ }^{8}(2002)$ showed that while several prognostic factors have been identified in breast carcinoma, the clinical outcome remains hard to predict for individual patients. Better predictive markers are needed to help guide difficult treatment decisions. the expression of cytokeratin 17 and/or cytokeratin 5 mRNAs. 2(5.6\%) patients were Grade I, 23(63.9\%) patients were Grade II and $11(30.6 \%)$ patient were Grade III. They found that expression of cytokeratin 17 and/or cytokeratin 5/6 in tumor cells was associated with a poor clinical outcome. Moreover, multivariate analysis showed that in node-negative breast carcinoma, expression of these cytokeratins was a prognostic factor independent of tumor size and tumor grade.

We showed that in POSITIVE study, 25(61.0\%) patient had PR status. In POSITIVE study, 10(24.4\%) patient had Her 2 neu. In POSITIVE study, 5(13.9\%) patient had HER2-positive (non-luminal), 1(2.8\%) patient had Luminal A-like, 19(52.8\%) patient had Luminal A-like, $3(8.3 \%)$ patient had luminal B-like HER 2+VE, 3(8.3\%) patient had Luminal B-like( HER2-NEG) and 5(13.9\%) patient had Triplenegative. In POSITIVE study, 6(14.6\%) patient had Cytokeratin 5/6. In above table showed that the mean Age (mean \pm s.d.) of patients was $50.8049 \pm 8.1216$. In above table showed that the mean Age in years (mean \pm s.d.) of patients was $2.4878 \pm .9519$. In above table showed that the mean Ki $67 \exp (\operatorname{mean} \pm$ s.d.) of patients was $.2122 \pm .2040$. In above table showed that the mean Ki 67 Grade (mean \pm s.d.) of patients was $1.5610 \pm .8077$. In above table showed that the mean Exp CK 5/6 (mean \pm s.d.) of patients was $.0585 \pm .1565$. In Positive Group, $2(33.3 \%)$ patients were $\leq 40$ years old, $2(33.3 \%)$ patients were $41-50$ years. old, $1(16.7 \%)$ patients were $51-60$ years old and $1(16.7 \%)$ patients were 61-70 years old. Association of Age in years vs group was not statistically significant $(\mathrm{p}=0.5479)$.

We found in Positive Group, 4(66.7\%) patients Left side and 2(33.3\%) patients were Right side. Association of side vs group was not statistically significant $(\mathrm{p}=0.1750)$. In Positive Group, $1(20.0 \%)$ patients T2N2MX, 3(60.0\%) patients T3N1MX and $1(20.0 \%)$ patients were T3N2MX. Association of pTNM vs group was statistically significant $(\mathrm{p}=0.0053)$. In Positive Group, 5(100.0\%) patients had S3 stage. Association of STAGE vs group was statistically significant $(\mathrm{p}=0.0014)$. In Positive Group, 3(50.0\%) patients had IBC-NST with medullary differentiation, 2(33.3\%) patients had Metaplastic CA and $1(16.7 \%)$ patients had UDH. Association of Histopathological Type vs group was statistically significant $(\mathrm{p}<0.0001)$. In Positive Group, $1(20.0 \%)$ patients had Grade I, $2(40.0 \%)$ patients had Grade II and $2(40.0 \%)$ patients had Grade III. Association of grade vs group was statistically significant $(\mathrm{p}=0.0324)$.

Otterbach F et al ${ }^{9}(2010)$ found that aquaporin1 (AQP1) is a water channel protein that facilitates water flux across cell membranes. In this study the immunohistochemical expression of AQP1 in 203 invasive breast carcinomas with long-term follow up was investigated. AQP1 expression was demonstrated in 11 tumours $(5.4 \%)$ and showed highly significant correlation with high tumour grade, medullary-like histology, "triplenegativity", cytokeratin 14 and smooth muscle actin expression. In univariate analysis, AQP1 was significantly associated with poor prognosis.

Makki $\mathrm{J}$ et al ${ }^{10}(2015)$ found that mammary carcinoma is the most common malignant tumor in women, and it is the leading cause of mortality, with an incidence of $\geq 1,000,000$ cases occurring worldwide annually. They exhibit a wide scope of morphological features, different immunohistochemical profiles, and unique histopathological subtypes that have specific clinical course and outcome. Breast cancers can be classified into distinct subgroups based on similarities in the gene expression profiles and molecular classification.

Our study showed that in Positive Group, 1(16.7\%) patients had ER status. Association of ER status vs group was statistically significant $(p=0.0007)$. Association of PR status vs group was statistically significant $(\mathrm{p}=0.0009)$. Association of Her 2 neu vs group was not statistically significant $(\mathrm{p}=0.1321)$. In Positive Group, $5(100.0 \%)$ patients had Triple-negative. Association of Molecular Subtypes vs group was statistically significant ( $\mathrm{p}=<0.0001$ ). In Positive Group, the mean Age (mean \pm s.d.) of patients was $48.5000 \pm 10.5972$. Difference of mean Age with both Group was not statistically significant $(\mathrm{p}=0.4588)$. In Positive Group, the mean Age in years (mean \pm s.d.) of patients was $2.1667 \pm 1.1690$. Difference of mean Age in years with both Group was not statistically significant $(\mathrm{p}=0.3778)$. In Positive Group, the mean Ki $67 \exp ($ mean \pm s.d.) of patients was $.5167 \pm .2927$. Difference of mean Ki 67 exp with both Group was statistically significant $(\mathrm{p}<0.0001)$. In Positive Group, the mean Ki 67 exp (mean \pm s.d.) of patients was $2.5000 \pm .8367$. Difference of mean Ki 67 Grade with both Group was statistically significant $(\mathrm{p}=0.0012$ ). In Positive Group, the mean Exp CK 5/6 (mean \pm s.d.) of patients was .4000 \pm .1789. Difference of mean Exp CK 5/6with both Group was statistically significant $(\mathrm{p}<0.0001)$.

\section{CONCLUSION}

We found that cytokeratin 5/6 expression in Breast Carcinoma was significantly associated with high grade of the tumour. cytokeratin 5/6 expression was significantly associated with advanced stage of the tumour. It was found that cytokeratin 5/6 expression was significantly correlated with ki67 in Breast Carcinoma patients. Hormonal receptor like ER and PR were was significantly associated with cytokeratin 5/6 expression tumour. Triple negative Breast Carcinoma patient was significantly associated with high grade of cytokeratin $5 / 6$ expression tumour. 
Table: Distribution of Cytokeratin 5/6, ER status and PR status.

\begin{tabular}{|c|c|c|c|}
\hline \multirow{3}{*}{ Cytokeratin 5/6 } & & Frequency & Percent \\
\cline { 2 - 4 } & Negative & 35 & $85.4 \%$ \\
\cline { 2 - 4 } & Positive & 6 & $14.6 \%$ \\
\hline \multirow{3}{*}{ ER status } & Total & 41 & $100.0 \%$ \\
\cline { 2 - 4 } & NEGATIVE & 11 & $26.8 \%$ \\
\cline { 2 - 4 } & POSITIVE & 30 & $73.2 \%$ \\
\cline { 2 - 4 } & Total & 41 & $100.0 \%$ \\
\hline \multirow{3}{*}{ PR status } & NEGATIVE & 16 & $39.0 \%$ \\
\cline { 2 - 4 } & POSITIVE & 25 & $61.0 \%$ \\
\cline { 2 - 4 } & Total & 41 & $100.0 \%$ \\
\hline
\end{tabular}

\section{REFERENCE}

1. Dr S Lakshmi Vidhya,DrRabiaSeyed. Expression of basal cytokeratinsin breast cancers. Global J.Pathol.Microbiol. 2017;17(2).

2. Mohammadizadeh Fereshteh, NaimiAzar, Parvin Rajabi, Ghasemibasir Hamidreza Expression of basal and luminal cytokeratins in breast carcinoma and their correlation with clinicopathological prognostic variables. Indian J Med Sci. 2009 Apr;63(4).

3. Buerger H, Otterbach F, Simon R, Poremba C, Diallo R, Decker T, Riethdorf L, Brinkschmidt C, Dockhorn-Dworniczak B, and Boecker W (1999b). Comparative genomic hybridization of ductal carcinoma in situ of the breast: Evidence of multiple genetic pathways. J Pathol 187:396-402.

4. Boecker W, Moll R, Poremba C, Holland R, van Diest PJ, Dervan P, Buerger H, Wai D, Diallo R, Herbst H, Schmidt A, and Buchwallow IB (2002). Common adult stem cells in the human breast give rise to glandular and myoepithelial cell lineages: A new cell biological concept. Lab Invest 82: 737-746.

5. Ku NO, Michie S, Resurreccion EZ, Broome RL, Omary MB Keratin binding to 14-3-3 proteins modulates keratin filaments and hepatocyte mitotic progression. Proc Natl proteins modulates keratin filaments and hepatocyte mitotic progression. Proc Natl
Acad Sci U S A 2002;99(7):4373-8.

6. Jana D, Mandal S, Mukhopadhyay M, Mitra D, Mukhopadhyay SK, Sarkar DK. Prognostic significance of HER-2/neu and survival of breast cancer patients attending a specialized breast clinic in Kolkata, Eastern India. Asian Pacific Journal of Cancer Prevention. 2012;13(8):3851-5.

7. Maeda I, Tajima S, Kanemaki Y, Tsugawa K, Takagi M. Use of immunohistochemical analysis of CK5/6, CK14, and CK34betaE12 in the differential diagnosis of solid papillary carcinoma in the breast. SAGE open medicine. 2018 Nov; 6:2050312118811542.

8. Fadare O, TavassoliFA. The phenotypic spectrum of basal-like breast cancers: a critical appraisal. Advances in anatomic pathology. 2007 Sep 1;14(5):358-73.

9. Van De Rijn M, Perou CM, Tibshirani R, Haas P, Kallioniemi O, Kononen J, Torhorst J, Sauter G, Zuber M, Köchli OR, Mross F. Expression of cytokeratins 17 and 5 identifies a group of breast carcinomas with poor clinical outcome. The American journal of pathology. 2002 Dec 1;161(6):1991-6.

10. Otterbach F, Callies R, Adamzik M, Kimmig R, Siffert W, Schmid KW, Bankfalvi A. Aquaporin 1 (AQP1) expression is a novel characteristic feature of a particularly aggressive subgroup of basal-like breast carcinomas. Breast cancer research and
treatment 2010Feb 1:120(1):67-76.

11. Makki J. Diversity of breast carcinoma: histological subtypes and clinical relevance. Clinical Medicine Insights: Pathology. 2015 Jan;8:CPath-S31563. 